\title{
AVALIAÇÃO DO ESTADO NUTRICIONAL E CONSUMO ALIMENTAR EM PACIENTES COM DOENÇA DE ALZHEIMER
}

Letícia Paranaíba MENDES*

Roberta Monterazzo CYSNEIROS**

Edeli Simioni de ABREU**

Daniela Maria Alves CHAUD**

\author{
*Nutricionista egressa do Curso de Nutrição do Centro de Ciências Biológicas e da Saúde - Universidade Presbiteriana \\ Mackenzie. \\ **Docentes do Centro de Ciências Biológicas e da Saúde - Universidade Presbiteriana Mackenzie. \\ Endereço eletrônico para correspondência: daniela.chaud@mackenzie.br
}

Recebido em: 18/11/2015 - Aprovado em: 13/08/2016 - Disponibilizado em: 18/12/2016

\begin{abstract}
RESUMO
A Doença de Alzheimer (DA) é a mais predominante dentre as principais demências. A síndrome clínica é uma doença neurodegenerativa que atinge $50 \%$ a $70 \%$ de todos os casos. Um estado nutricional inadequado pode afetar as condições gerais e de bem estar do paciente. O problema nutricional mais prevalente é a perda de peso, podendo levar a desnutrição, ocorrendo insidiosamente em todos os estágios da doença. Esta perda de peso está relacionada com a agitação, processos infecciosos e distúrbios neurológicos provenientes da doença, que levam a redução da ingestão de alimentos. O objetivo deste trabalho foi avaliar o estado nutricional e consumo alimentar de pacientes com doença de Alzheimer em uma casa de repouso no município de São Paulo. Foram avaliados 28 indivíduos $(\geq 65$ anos $\pm 5,75)$, sendo 14 com DA e 14 indivíduos sadios, considerados do grupo controle (GC). As informações sócio demográficas e fatores influenciadores na DA foram avaliadas pelo inquérito clínico. O consumo alimentar pelo recordatório de 24 horas e o estado nutricional foi avaliado pela aplicação da Mini Avaliação Nutricional (MAN). O IMC médio (calculado a partir do peso e altura referidos) foi de 19,95 \pm 6,54 (DA) e 23,8 \pm 5,96 (GC). O risco de desnutrição foi de $50 \%$ em ambos os grupos, já a desnutrição foi evidenciada somente no grupo DA. A dificuldade na deglutição foi observada no grupo DA. A ingestão calórica do grupo controle obteve um déficit $(35,6 \%)$ ocorrendo o mesmo quanto ao consumo de carboidratos $(73,3 \%)$, proteínas $(65,5 \%)$ e lipídeos $(35,6 \%)$. O consumo de ácido fólico, vitamina A, E, B12, B6, D e fibras foi maior no grupo DA. Desta forma, observamos a importância do acompanhamento com o profissional nutricionista e médico, visando o diagnóstico precoce da doença como também o nutricional com o intuito de reverter e/ou prevenir os agravos nutricionais da DA.
\end{abstract}

Palavras chave: Doença de Alzheimer. Desnutrição. Idosos.

\begin{abstract}
Alzheimer's disease (AD) is the most prevalent among the main dementias, reaching $50 \%$ to $70 \%$ of all cases. An inadequate nutritional status can affect the overall condition and well being of the patient. The most prevalent nutritional problem is weight loss, leading to malnutrition, occurring at all stages of disease. This weight loss is related to the stirring, infectious processes and neurological disorders arisen from the disease, that leads to reduced food intake. The purpose of this article is to evaluate the nutritional status and dietary intake of patients with Alzheimer's disease in a nursing home in the city of São Paulo. Were evaluated a total of 28 people ( $\geq 65$ years \pm 5.75$), 14$ with Alzheimer's disease (AD) and 14 healthy considered the control group (CG). Sociodemographic information and influencing factors in Alzheimer were evaluated by clinical investigation. Food consumption by 24- hour recall and nutritional status by applying the Mini Nutritional Assessment (MNA). The mean BMI (calculated from weight and height) was $19.95 \pm$ 6.54 (DA) and $23.8 \pm 5.96$ (GC). The risk of malnutrition was 50\% in both groups, since malnutrition only in the AD group. The GC caloric intake déficit scores (35.6\%) occurring at the same consumption of carbohydrates (73.3\%) protein $(65.5 \%)$ and lipids (35.6\%). The use of folic acid, vitamin A, E, B 12 , B 6 , D and fibers was higher in the AD group. Thus, we observed the importance of following up with the professional nutritionist and doctor in order to reverse and / or prevent nutritional problems of AD.
\end{abstract}

Key words: Alzheimer's disease. Malnutrition. Elderly. 


\section{INTRODUÇÃO}

Com o avançar da tecnologia e novas descobertas na área da saúde, ocorreu o aumento de idosos no mundo. No Brasil, o aumento da proporção de idosos começou a ser verificado em 1960 e vem ocorrendo de maneira muito rápida, com estimativas apontando a proporção de idosos (com 65 anos ou mais) deverá aumentar de 5,1\% em 2000 para 14,2\% em 2050 (CHAIMOWICZ, 1998).

A nutrição é um aspecto importante no contexto das mudanças fisiológicas relacionados a idade e desenvolvimento de doenças crônicas não transmissíveis, como diabetes mellitus, doenças cardiovasculares e obesidade, importantes fatores de risco no desenvolvimento da Doença de Alzheimer (DA) (TAVARES; ANJOS, 1999).

O estado nutricional inadequado pode afetar as condições gerais e de bem estar do paciente influenciando na evolução da demência. Devido os transtornos neurológicos graves comuns que alteram os mecanismos e as capacidades cognitivas e motoras, necessárias para uma alimentação adequada, o estado nutricional pode ser afetado (MUÑOZ; AGUDELO; LOPERA, 2006).

Os problemas nutricionais prevalentes nas demências são a perda de peso, fator de risco para a desnutrição, bem como a desidratação. Tais condições tornam os indivíduos suscetíveis a infecções, agravamento da doença e diminuição acentuada na ingestão de alimentos e consequentemente de calorias e nutrientes(MAGALDI;

MORILLO;

KIKUCHI, 2006; ALVARENGA, 2009).

Pacientes com demência necessitam de um aumento calórico devido algumas alterações e agitação, porém, com o avanço da doença, ocorre uma mudança do hábito alimentar, aumentando a ingestão de carboidratos simples e a diminuição de proteínas, devido a dificuldade na mastigação e deglutição que pode acometer estes pacientes (SAMPAIO; SABRY, 2007).

Em 1987, Veras; Ramos e Kalache já abordavam o crescimento da população idosa no Brasil, o que refletia a melhoria nas condições de vida.

Inúmeras são as causas da demência, cujo diagnóstico depende das diferentes manifestações clínicas apresentadas pelo indivíduo. O principal fator de risco é a idade (ANS, 2009).

A partir do acompanhamento do caso de uma de suas pacientes,Sra. Auguste Deter, do hospital onde trabalhava, Alois Alzheimer relatou pela primeira vez no $37^{\circ}$ Congresso do Sudoeste da Alemanha de Psiquiatria uma patologia neurológica com demência, alterações no comportamento e incapacidade para atividades rotineiras. Mais tarde descreveu a doença no ponto de vista 
patológico, sobre as placas senis e os novelos neurofibrilares (HIPPIUS; NEUNDÖRFER, 2003), posteriormente a entidade clínica fora nomeada com o nome do pesquisador que a relatou pela primeira vez.

A Doença de Alzheimer (DA) é uma demência que inclui deterioração progressiva de habilidades intelectuais e rotineiras e declínio cognitivo.Atinge na maioria dos casos, indivíduos com 65 anos ou mais, porém em alguns pode se manifestar mais precocemente (CAIXETA, 2012).

Atinge homens e mulheres, sendo considerada uma doença do envelhecimento por acometer pessoas com 60 anos ou mais, sendo chamada nesta forma de senil ( $90 \%$ dos casos), porém raramente (5\%) são os casos de indivíduos após os 40 anos de idade, a forma pré-senil por recorrência familiar com um comportamento mais agressivo por ser progressivo o comprometimento cognitivo (MAHAN; STUMP, 2005).

Muitas vezes são aquelas em que o indivíduo e até a família trata a doença como sintoma do envelhecimento, sendo descoberta tardiamente, dificultando o tratamento e/ou manutenção da memória dos pacientes (ABRAZ, 2013).

Através dos exames de neuroimagem, torna-se possível visualizar a atrofia cortical difusa presente em pessoas com DA, como também a presença de grande número de placas senis e novelos neurofibrilares, degenerações grânulo-vacuolares e perda neuronal. Além da atrofia característica da doença, o acúmulo da proteína b-amilóide nas placas senis e da microtubulina tau nos novelos neurofibrilares, são aspectos importantes no grau de demência nos afetados (NITRINI et al.,2005).

Acreditam que seja improvável ser originada de apenas uma única causa, sendo assim, o conjunto de fatores pode conduzir ao desencadeamento, tendo sua etiologia multifatorial, ou seja, a associação de fatores genéticos e ambientais levam a perda cognitiva e manifestações clínicas da doença (MAHAN; STUMP, 2005).

Alguns fatores estão sendo descritos recentemente, como os socioeconômicos, estilo de vida e clínicos que englobam o baixo nível de escolaridade e renda, associação com doenças cardiovasculares, diabetes mellitus, sedentarismo e hábitos alimentares inadequados (LOPES et al., 2011).

A medida que a idade avança, maior a probabilidade da ocorrência de DA. Evidências bioquímicas demonstram que a cadeia de transporte mitocondrial está afetada na DA, ajudando no declínio da memória e dos processos de aprendizagem (SERENIKI; VITAL, 2008).

A literatura aponta que a DA é mais prevalente em mulheres, podendo ressaltar 
sobre a predominância da população idosa ser feminina, provavelmente devido ao fato de ser mais longeva e, portanto, vulnerável ao desenvolvimento à DA (NITRINI, 1999).

O fator genético é considerado atualmente como fator preponderante na etiopatogenia da DA, mesmo que na enfermidade,é estabelecido o acúmulo de eventos genéticos e ambientais que contribuem com pequenos efeitos que, em conjunto, resultam no estabelecimento da doença com o grau de severidade (SERENIKI; VITAL, 2008).

A apolipoproteína E (ApoE)é uma das principais proteínas presentes no plasma humano, localizada no cromossomo 19, para onde é exportada após sua síntese em diversos órgãos, especificamente no fígado, sendo a principal glicoproteína encontrada no cérebro (OJOPI; BERTONCINI; NETO, 2004).

A glicoproteína apresenta três alelos, $\varepsilon 2, \varepsilon 3, \varepsilon 4$, aumentando até cinco vezes a probabilidade de desenvolvimento da doença quando há a herança de um ou dois desses alelos. O fator genético mais comum para a DA é a identificação do alelo polimórfico ¿4do gene ApoE, representado em excesso nos indivíduos com a doença, comparados com a população em geral (FRIDMAN et al., 2004).

Em 1993, foi descrito o primeiro estudo, de Corder et al., demonstrou que o risco da DA conferido pelo alelo ApoE4 aumenta de forma dose-dependente, ou seja, o risco da DA aumenta de $20 \%$ a $90 \%$, e a média de idade de início da doença diminui de 84 para 68 anos com o aumento no número de alelos.

Estudos mostram a ApoE com o papel específico na formação de placas amiloides e amaranhados neurofibrilares. Além de desempenhar um papel fundamental no catabolismo de componentes ricos em triglicérides no corpo, podendo causar inúmeras doenças envolvidas com o aumento no nível de colesterol e triglicérides na circulação na deficiência dessa proteína, devido ao não reconhecimento dessas moléculas pelos receptores de membrana dos quilomícrons e pelas moléculas de VLDL, responsáveis por sua captação no fígado (OJOPI; BERTONCINI; NETO, 2004).

Conhecida como APP, a proteína percursora de amiloide, quando fragmentada, origina partículas denominadas $\beta$-amilóide (A $\beta)$ de 40 a 42 aminoácidos, sendo neurotóxico e seu acúmulo resulta na formação de fibras amiloides, formando placas senis (THOMAZ; VIEBIG, 2012; FRIDMAN et al., 2004).

As presenilinas 1 (PSEN1) e presenilinas 2 (PSEN2) são proteínas cujas mutações estão relacionadas a alteração na fragmentação da APP, aumentando a produção de $\mathrm{A} \beta$ e originando casos de Alzheimer. Mutações na PSEN1 e PSEN2 são responsáveis por apenas 
$30 \%$ a $50 \%$ dos casos de herança dominante e início precoce (FRIDMAN et al., 2004).

Nas mutações do gene PSEN1 ocorre a associação no processo inflamatório observado na placa amiloide e pode interferir no processo de apoptose, já na PSEN2, pode aumentar a velocidade do processo de neurodegeneração (THOMAZ; VIEBIG, 2012).

Tendo em vista as premissas supracitadas, o objetivo desse trabalho foi avaliar o estado nutricional e consumo alimentar de mulheres com doença de Alzheimer.

\section{METODOLOGIA}

Trata-se de um estudo de caráter transversal com coleta de dados primários, sendo classificada quanto a sua natureza como aplicada, descritiva, com abordagem qualitativa e quantitativa em um período de tempo.

A amostra escolhida se refere a 30 indivíduos com idade superior a 65 anos, sendo 14 portadores da Doença de Alzheimerfrequentadores de uma casa de repouso na cidade de São Paulo e 14 indivíduos sem o diagnóstico da doença, frequentadores de uma Igreja na cidade de São Paulo.

Foram incluídas na pesquisa pessoas diagnosticadas clinicamente com Doença de Alzheimer e idade maior ou igual a 65 anos, como também indivíduos que não possuem nenhum tipo de demência e idade maior e igual a 65 anos.

A população estudada foi do sexo feminino e que aceitaram voluntariamente o convite para participar da pesquisa.

Os indivíduos com DA foram diagnosticados anteriormente a partir de uma avaliação clínica e a realização de testes como: mini teste de exame de estado mental e o teste do relógio, sendo confirmados em ressonância magnética e tomografia computadorizada.

Foram excluídos indivíduos com idade menor a 65 anos e/ou que não aceitaram participar da pesquisa.

Para a coleta de dados foram realizadas entrevistas com os indivíduos indivíduos que se adequaram aos critérios de inclusão. Quando na impossibilidade de responder, foi questionado aos cuidadores.

As entrevistas incluíram a coleta de informações sócio demográficas e fatores influenciadores no Alzheimer pelo inquérito clínico, a aplicação da Mini Avaliação Nutricional (MAN) e o Recordatório de 24 horas (R24), após a assinatura do termo de consentimento livre e esclarecido e carta de informação ao sujeito de pesquisa.

A coleta de dados foi feita pela pesquisadora onde a mesma foi aos 
estabelecimentos frequentados pelos indivíduos e cuidadores que participaram da pesquisa.

O inquérito clínico foi desenvolvido com o objetivo de obter maiores características de cada indivíduo que participou da pesquisa. $\mathrm{O}$ inquérito é composto por quesitos que foram respondidos pelos entrevistados, ou quando na impossibilidade, pelos cuidadores.

A classificação para a circunferência da cintura foi realizada através da obtenção do valor da medida da circunferência da cintura, a qual foi realizada com a pessoa em pé e com o auxílio da fita métrica foi circundado o indivíduo no ponto médio entre a última costela e a crista ilíaca. Esses valores foram classificados de acordo com o proposto pela OMS em 1998 (BUSNELLO, 2007).

Para avaliar o estado nutricional dos pacientes, foi aplicado a Mini Avaliação Nutricional (MAN), a qual incluiu as seguintes medidas antropométricas: peso, altura, índice de massa corporal (IMC) classificado posteriormente segundo OPAS (2002), circunferência do braço (CB) e circunferência da panturrilha. A pesagem do indivíduo foirealizada a partir da balança OMRON HBF-516B e quando na impossibilidade, foiencontrada pela equação de Chumlea (1985), em que se utiliza valores de $\mathrm{CB}$, circunferência da panturrilha $(\mathrm{CP})$, prega cutânea subscapular (PCSe), e a altura do joelho (AJ). Estes dados são colocados na seguinte fórmula de acordo com o gênero feminino:

Mulheres $=[(1,27 \times \mathrm{CP})+(0,87 \times \mathrm{AJ})+$ $(0,98 \times \mathrm{CB})+(0,4 \times \mathrm{PCSe})-62,35]$

Para a obtenção de valores para a estatura do entrevistado, foi utilizado o estadiômetro com medidas em centímetros. Sendo realizada com a pessoa em pé, descalço, com os calcanhares unidos, costas e cabeça eretas e braços estendidos ao lado do corpo. $\mathrm{Na}$ impossibilidade de obter esta medida, foi utilizado valores estimados através da fórmula de Chumlea, 1985, em que se utiliza o valor de $\mathrm{AJ}$ e a idade em anos.

Homens: Altura $(\mathrm{cm})=64,19-(0,04 \mathrm{x}$ idade em anos $)+(2,02 \mathrm{x}$ altura do joelho em $\mathrm{cm})$

Mulheres: Altura $(\mathrm{cm})=84,88-(0,24 \mathrm{x}$ idade em anos $)+(1,83 \mathrm{x}$ altura do joelho em $\mathrm{cm})$

Para aferição das medidas das circunferências corporais foi utilizado uma fita métrica inelástica.

O Recordatório de 24 horas, ou seja, o inquérito alimentar que foi utilizado, objetivou criar bases metodológicas para verificar a adequação da ingestão de alimentos e seus nutrientes em um dia habitual. 
O consumo alimentar avaliado por meio de R24h de um dia habitual foi aplicado com $\mathrm{o}$ intuito do relato de todos os alimentos e bebidas consumidos no dia anterior à entrevista, sendo também questionados sobre o horário, local e medidas caseiras.

Após a aplicação do inquérito alimentar, foi realizada análise da densidade energética da dieta e os seguintes nutrientes: carboidratos, proteínas, lipídeos, fibra alimentar, ácido fólico, vitaminas A, E, B $12, B_{6}$ e D.

Os recordatórios foram analisados individualmente $\mathrm{e}$ as medidas caseiras descritas pelos pacientes convertidas em gramas e mililitros pela pesquisadora. A análise da composição centesimal dos alimentos foi realizada com auxílio do programa Avanutri ${ }^{\circledR}$, consideradas as Dietary Reference Intakes- DRI (2002) para idade e sexo.

Os dados foram tabulados no programa Microsoft Excel® 2010 e os resultados apresentados sob a forma de tabelas e gráficos, segundo suas distribuições percentuais, média, desvio-padrão e valor p.

Por se tratar de pesquisa com seres humanos, o projeto foi submetido ao Comitê de Ética em Pesquisa da Universidade Presbiteriana Mackenzie. Os sujeitos foram informados sobre as etapas e os procedimentos do estudo e assinaram um Termo de Consentimento Livre e Esclarecido para a autorização expressa da utilização dos dados, garantindo-lhes anonimato e confidencialidade em todas as etapas da pesquisa.

$\mathrm{O}$ estudo segue as diretrizes e normas que regulamentam as pesquisas envolvendo seres humanos presentes no código de bioética e na legislação brasileira (Resolução 196/96).

O projeto em questão atende à aprovação pelo Comitê de Ética em Pesquisa da Universidade Presbiteriana Mackenzie, conforme protocolo CIEP $\mathrm{n}^{\circ} \mathrm{N} 011 / 04 / 13$.

\section{RESULTADOS}

Os estágios da doença de Alzheimer analisados foram de leve $(n=6)$ a moderado $(\mathrm{n}=8)$ com um tempo médio de 4 anos desde o aparecimento da doença.

A análise das variáveis antropométricas e da aplicação do MAN se encontram na tabela 1 e gráfico 1. A idade média estudada do grupo controle (CG) foi de 75 anos com o IMC aceitável $\left(23,8 \mathrm{~kg} / \mathrm{m}^{2}\right)$, porém com $28 \%$ $(n=4)$ desta população classificadas como desnutridos, segundo OPAS (2002). No grupo de pessoas com Doença de Alzheimer (DA) a idade média estudada foi 83 anos com IMC médio de $19,9 \mathrm{~kg} / \mathrm{m}^{2}$, também classificados como desnutridos. 
Tabela 1 -Distribuição de características antropométricas e da composição corporal do grupo controle e de portadores. São Paulo, 2015.

\begin{tabular}{lccc}
\hline Medidas & GC & DA & Valor p \\
& Média e DP & Média e DP & \\
\hline Idade (anos) & $75,00 \pm 6,22$ & $83,14 \pm 7,96$ & 0,006 \\
Peso atual $(\mathrm{kg})$ & $61,01 \pm 13,06$ & $49,76 \pm 18,07$ & 0,073 \\
Estatura $(\mathrm{m})$ & $1,58 \pm 0,08$ & $1,57 \pm 0,07$ & 0,888 \\
IMC $\left(\mathrm{kg} / \mathrm{m}^{2}\right)$ & $23,8 \pm 5,96$ & $19,95 \pm 6,54$ & 0,119
\end{tabular}

Devido impossibilidades, a medida da média de $93 \mathrm{~cm}$, o que está acima do circunferência da cintura no grupo DA não foi preconizado,aumento as chances do aferida, porém no CG foi observada uma aparecimento de doenças cardiovasculares.

Gráfico 1 - Comparação do resultado do MAN no grupo controle e no grupo de indivíduos com Doença de Alzheimer. São Paulo, 2015.

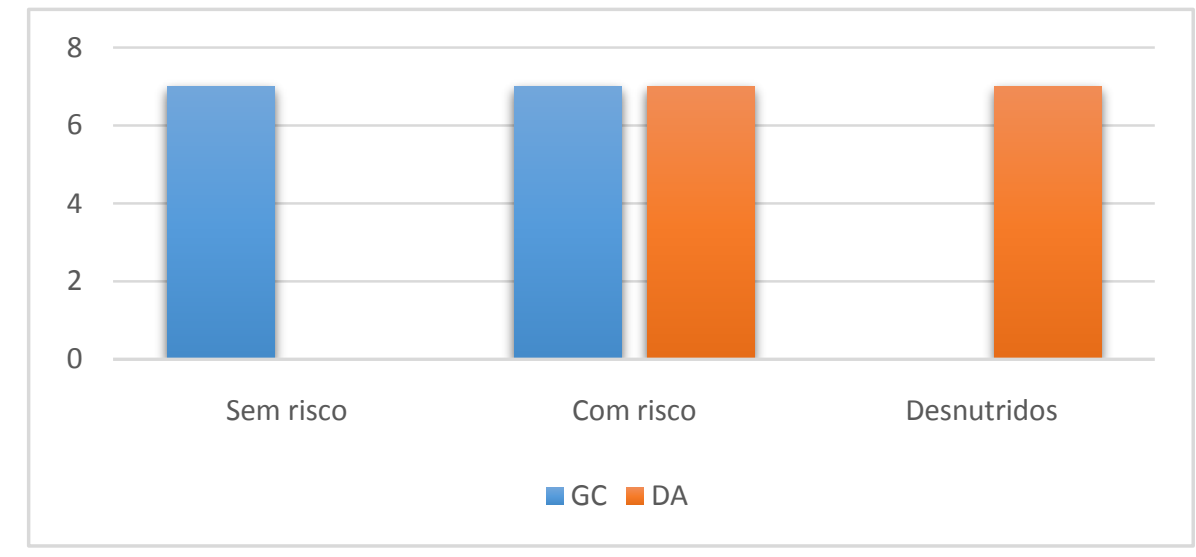

Teste do qui-quadrado: valor $\mathrm{p}<0,001$.

Nos dois grupos a maioria dos indivíduos se exercitam, porém, as atividades encontradas são diferentes, sendo, no GCpraticantes de aula de dança e caminhada, em sua maioria e no grupo DA ocorre devido às sessões de fisioterapia.

Em relação à dificuldade na deglutição, o grupo DA obteve mais casos $(n=6)$, onde os mesmos fazem uso de espessantes na dieta.
A exposição solar como um importante fator para a síntese de vitamina D também obteve resultados satisfatórios, onde os dois grupos exercem diariamente. Apenas dois indivíduos do grupo DA e um do GC não se expõem devido a enfermidades e/ou impossibilidades. 
Gráfico 2 - Distribuição dos resultados encontrados pelo inquérito clínico no grupo controle e de portadores. São Paulo, 2015.

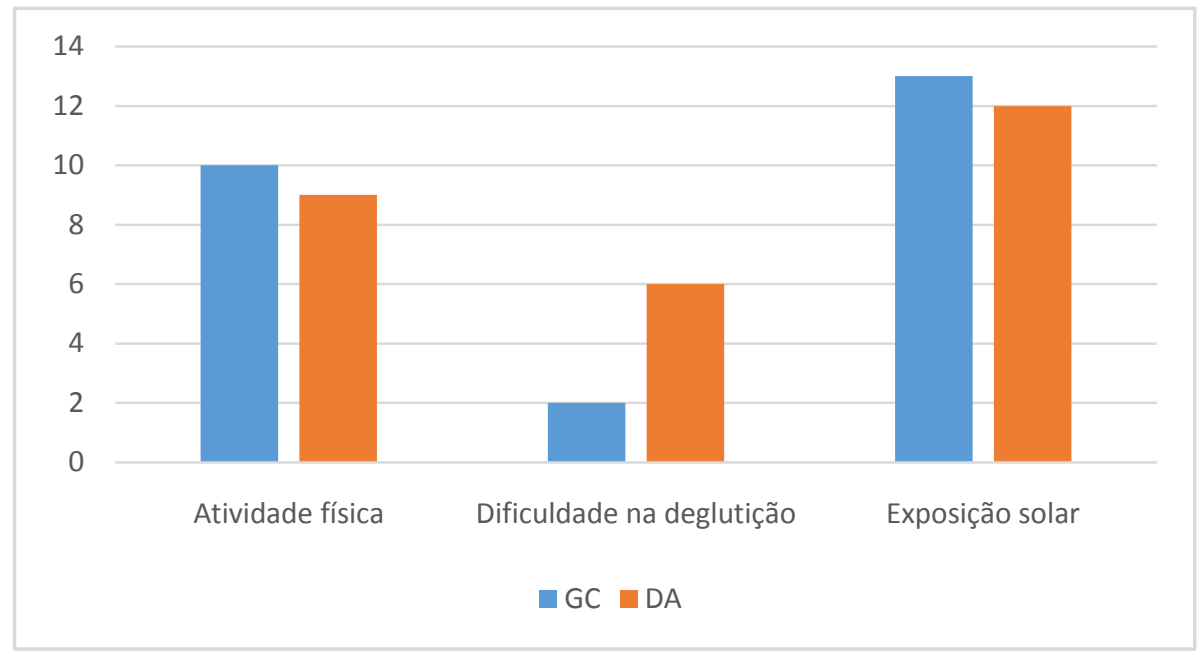

Teste do qui-quadrado: valor $\mathrm{p}<0,364$

O grupo DA faz, em sua maioria $(\mathrm{n}=12)$, o consumo de medicamentos durante as refeições constituídas de dietas prescritas por nutricionista, já no GC foi encontrado apenas um indivíduo que realiza o ato de seguir uma dieta recomendada por médico e um a realizar a medicação durante a refeição.

A ingestão calórica do GC obteve um déficit de 35,6\% em relação ao grupo DA. O mesmo ocorreu na ingestão de macronutrientes do GC, onde o consumo de carboidratos foi de $73,3 \%$, proteínas $65,6 \%$ e lipídeos 35,6\%a menos em comparação com o grupo DA, porém é importante ressaltar que quatro indivíduos estudados fazem uso de espessantes devido a disfagia, aumentando assim o valor calórico e consumo de carboidratos.

Tabela 2 - Distribuição percentual de adequação da ingestão de fibras alimentares e micronutrientes, 2013.

\begin{tabular}{ccccc}
\hline Nutrientes & CG & DA & Recomendação & Valor p \\
\hline Fibras $(\mathrm{g})$ & $9,61 \pm 4,89$ & $25,86 \pm 9,28$ & $20 \mathrm{~g}-30 \mathrm{~g}$ & $<0,001$ \\
Ácido fólico $(\mathrm{mg})$ & $47,93 \pm 45,84$ & $68,27 \pm 26,21$ & 400 & 0,161 \\
Vitamina A (mg) & $158,86 \pm 134,28$ & $1044,07 \pm 308,63$ & 700 & $<0,001$ \\
Vitamina E (mg) & $6,16 \pm 6,24$ & $11,68 \pm 3,82$ & 15 & 0,009 \\
Vitamina B $12(\mathrm{mg})$ & $1,74 \pm 1,60$ & $2,53 \pm 1,17$ & 2,4 & 0,148 \\
Vitamina B $(\mathrm{mg})$ & $0,39 \pm 0,14$ & $1,11 \pm 0,41$ & $1,5-1,7$ & $<0,001$ \\
Vitamina D $(\mathrm{mg})$ & $1,80 \pm 2,99$ & $2,18 \pm 2,11$ & 15 & 0,704 \\
\hline
\end{tabular}


Devido à dieta individualizada prescrita e acompanhada pela nutricionista do local de estudo do grupo DA, o consumo de nutrientes, como um todo, foi maior. Vale ressaltar que, seis pessoas fazem uso desuplementos nutricionais via oral que são adicionados às refeições. Dentre essas, três indivíduos fazem uso de alimentação nutricional via enteral visando a melhoria do estado clínico geral, principalmente o nutricional.

Podemos supor assim, o porque da grande diferença encontrada após a realização do valor $\mathrm{p}$, na ingestão de fibras, vitamina $\mathrm{A}$ e $B_{6}$, nutrientes que se encontram em alto teor em suplementos alimentares.

\section{DISCUSSÃO}

Dentre os dois grupos avaliados, 50,0\% $(\mathrm{n}=14)$ apresentaram risco nutricional segundo a Mini Avaliação Nutricional (MAN). Este resultado é compatível com o estudo realizado por Souza et al. (2012) que encontrou maior prevalência de risco de desnutrição em portadores de Alzheimer também através do MAN.

Os idosos apontados como desnutridos pela MAN também foram classificados como baixo peso pelo IMC. Desta forma, a Mini Avaliação Nutricional (MAN) é um procedimento eficiente e preciso para determinar um escore indicador de desnutrição, sendo um método mais sensível em relação ao IMC devido a sua capacidade em estimar perda de peso e capacidade funcional através de questões e medidas antropométricas (MAHAN; STUMP, 2005; VELLAS M. et al., 1999).

O achado de $25 \%$ do GC com desnutrição, segundo a aplicação do MAN, é semelhante ao estudo de Souza et al.(2012) em que foi observado a incidência de $25 \%$ dos estudados com desnutrição através do MAN.

Segundo os resultados, mesmo com o alto consumo energético, de macronutrientes, fibras e micronutrientes no grupo DA, o mesmo obteve IMC abaixo do preconizado e maior índice de desnutrição em relação ao GC. Pode-se sugerir a hipótese de que este resultado se deve ao maior gasto calórico de pessoas com DA, pela agitação, alterações no organismo e neurodegeneração, mesmo com a suplementação, a oferta não atinge o gasto energético.

Estimativas de 2011, nos Estados Unidos da América, mostraram que 5,4 milhões de americanos, de todas as idades, possuem DA e outras demências. Dentre estes, 5,2 milhões possuem idade de 65 ou mais anos, sendo a maioria, 3,4 milhões, do sexo feminino. Podendo, essa larga proporção de idosas que possuem a DA e outras demências, explicar o fato das mulheres viverem mais do que os homens (GOES, 2012). 
O próprio envelhecimento contribui para a redução da capacidade de realizar atividades e de se manter independente. Contudo, importante ressaltar a presença de ambos os grupos na atividade física, mesmo que em forma de caminhadas diárias ou de fisioterapia semanal.

Diferentemente de estudos já realizados, como o de Silva et al (2011),sobre a Doença de Alzheimer, os indivíduos com DA estudados neste trabalho não sofrem problemas com a mastigação, apenas na deglutição, facilitando a oferta de proteínas, nutriente que, pela dificuldade na mastigação se torna escasso, aumentando a incidência da desnutrição. Já em relação a deglutição, não causa grandes desconfortos, vendo que os indivíduos têm acesso a espessantes, facilitando a ingestão de todas as refeições.

Vale ressaltar que, além da DA analisada no estudo, indivíduos de ambos grupos apresentaram doenças como hipertensão arterial sistêmica, diabetes mellitus, síndrome das pernas inquietas e depressão. Este achado também foi o de Machado et al. (2009) onde observou uma predominância de hipertensão em $52 \%$ dos idosos.

Devido a ampla diferença em relação a ingestão de carboidratos, proteínas, lipídeos e fibras alimentares entre o GC e DA, não foi encontrado semelhança com nenhum estudo já publicado, maiores pesquisas se tornam necessárias.
O maior consumo de micronutrientes no grupo DA foi elevado em relação ao GC, este fato se deve à utilização da suplementação via oral e enteral, priorizando a intervenção e acompanhamento de um profissional nutricionista neste estágio da vida, o idoso sendo, ou não, portador de DA.

Os dados sobre as vitaminas não são compatíveis com o encontrado de Machado et al. (2009) em que se observou o déficit de vitamina $\mathrm{A}, \mathrm{E}, \mathrm{D}$ e $\mathrm{B}_{6}$ em idosos portadores de DA.

Mesmo com diferenças significantes entre os grupos, o consumo de micronutrientes e fibras de ambos grupos foram inadequados se comparados com a recomendação.

O consumo insatisfatório de vitamina D pode ser corrigida pelo resultado sobre a exposição solar, onde, a mesma é responsável por cerca de $90 \%$ da aquisição de vitamina D, porém, o uso de suplemento oral ou o aumento do consumo de alimentos fonte desta vitamina se torna necessário, já que é responsável pela saúde dos ossos, não os enfraquecendo, acontecimento frequente em idosos (SARAIVA et al., 2007).

Destaca-se que, as deficiências de ácido fólico e vitamina $B_{12}$ e $B_{6}$, resultam no aumento dos níveis de homocisteína, um fator de risco para doenças cardiovasculares e acidente vascular cerebral, podendo estar relacionada com um risco mais elevado de 
DA. Assim, a suplementação destes micronutrientes pode diminuir o risco de doença cardiovascular, acidente vascular cerebral e demência, reduzindo a homocisteína (SESHADRI et al., 2002).

Por mais que o grupo DA tenha consumido mais vitamina $\mathrm{E}$, em ambos os grupos foi verificado o déficit da mesma. A vitamina $\mathrm{E}$ pode ser manipulada como um tratamento eficaz da DA. No entanto, o efeito desta em pacientes com DA mostra variações consideráveis na sua função antioxidante e na sua capacidade de melhorar as funções cognitivas. Os níveis elevados dessa vitamina estão associados com o risco reduzido de DA na idade avançada (80 anos ou mais).

A menor ingestão de fibras alimentares no GC ocorreu devido ao baixo consumo de frutas e verduras observado através do R24 e o mesmo acorreria ao grupo DA caso não houvesse a suplementação nutricional, pois, se analisarmos o R24 deste grupo, sem a suplementação, o consumo de fibras seria inferior à recomendação.

Alguns medicamentos utilizados na DA têm como consequência a constipação, fazendo necessário o consumo de fibras alimentares, amenizando este desconforto.

Os fármacos utilizados nas doenças neurodegenerativas como a DA, o tratamento é basicamente medicamentoso, portanto, a necessidade de energia, macronutrientes e micronutrientes deve ser ajustada evitando ou minimizando as complicações que o tratamento proporciona além de haver diminuição dos sintomas provocados pela doença (REIS, 2004).

Idosos possuem perda de massa muscular devido ao metabolismo e funções orgânicas alteradas. A perda de peso, que está associada à deficiência de nutrientes causada pela baixa ingestão alimentar, pode gerar uma subnutrição. O mesmo ocorre com indivíduos portadores da doença de Alzheimer que apresentam distúrbios, comprometendo gravemente seu estado nutricional.

Com a evolução da doença, sabe-se que há significativa diminuição de peso. Além do gasto energético superior nos indivíduos com Doença de Alzheimer, o que leva à necessidade de uma correção no consumo alimentar para as necessidades energéticas adicionais (PALHAGEN et al., 2005).

\section{CONCLUSÃO}

Os fatos encontrados neste estudo sobre o consumo alimentar e estado nutricional de pessoas portadoras de DA se tornam interessantes para o aumento de pesquisas sobre o assunto, como também para abranger o conhecimento sobre os idosos e as diversas doenças que o corpo humano está suscetível a manifestar pela rotina diária e de hábitos alimentares e cotidianos não saudáveis. 
Em conclusão, a perda de massa muscular, vista como desnutrição apontada pela MAN em indivíduos com Doença de Alzheimer nos salienta a importância do acompanhamento com o profissional nutricionista e médico visando o diagnóstico da doença como também o nutricional precocemente, com o intuito de reverter e/ou prevenir os agravos nutricionais da Doença de Alzheimer.

\section{REFERÊNCIAS}

ALVARENGA, M. Saúde mental e nutrição: Mental health and nutrition. Nutrição em Pauta. v.17, n.96, p.16-22, 2009.

ANS - AGÊNCIA NACIOAL DE SAÚDE.

Manual técnico de promoção da saúde e prevenção de riscos e doenças na saúde suplementar / Agência Nacional de Saúde

Suplementar (Brasil). - 3. ed., 2009.

\section{BUSNELLO, F. M. Aspectos nutricionais} no processo do envelhecimento. Atheneu, 2007.

CHUMLEA, W. C., ROCHE, A. F., STEINGAUGH, M. L.Estimating stature from knee hejght for persons 60 to 90 years of age. J. Am. Geriatr. Soc., v. 33, p. 116 120, 1985.

CORDER, E.H. et al.Gene dose of apolipoprotein E type 4 allele and the risk of Alzheimer's disease in late onset families.

Science 261, p. 921-923, 1993.

\section{CAIXETA, L. Doença de Alzheimer.}

Artmed, 2012.
CHAIMOWICZ, F. Os idosos brasileiros no século XXI: demografia, saúde e sociedade. Postgraduate, 1998.

FRIDMAN, C. et al.Alterações genéticas na doença de Alzheimer. Rev. Psiquiatr., v. 31, n. 1, p. $19-25,2004$.

GOES, V. F. Avaliação Nutricional e Cognitiva de Pacientes com Diagnóstico Clínico da Doença de Alzheimer. Unicentro - PR, Guarapuava, 2012.

HIPPIUS H, NEUNDÖRFER G. The discovery of Alzheimer's disease. Dialogues Clin Neurosci. 2003;5:101-108.

LOPES, L. C. et al.Doença de Alzheimer: Prevenção e Tratamento. Diretrizes Clínicas na Saúde Suplementar, Associação Médica Brasileira e Agência Nacional de Saúde Suplementar, 2011.

MACHADO, J. et al.Estado nutricional na Doença de Alzheimer. Rev. Assoc. Med. Bras. v. 2, n. 55, p. $188-191,2009$.

MAGALDI, R. M.; MORILLO, L. S.; KIKUCHI, E. L. Comorbidades em demência. Revista Racine. v. 16, n. 95, p. 44-52, 2006.

MAHAN, L. K.; STUMP, S. S. Krause: Alimentos, Nutrição e Dietoterapia. São Paulo: Rocca, 2005, p. 304-323.

MUÑOZ, A. M., AGUDELO, G. M., LOPERA, F. J. Diagnóstico Del estado nutricional de los pacientes con demência tipo Alzheimer registrados en el Grupo de Nurociencias, Medelin, 2004. Revista Biomédica. Bogotá, v. 26, n. 1, mar, 2006. Disponível em: http://www.scielo.org.co/scielo.php?pid=S01 $2041572006000100014 \&$ script $=$ sci_arttext\&tl ng=es. Acessado em: outubro de 2014.

NITRINI, R., et al. Diagnóstico de Doença de Alzheimer no Brasil: Avaliação cognitiva e funcional. Recomendações do Departamento Científico de Neurologia Cognitiva e do Envelhecimento da Academia Brasileira de Neurologia. Arq. Neuropsiquiatr., v. 63, n. 3-A, p. $720-727,2005$. 
NITRINI, R. Epidemiologia da doença de Alzheimer no Brasil. Rev. de Psiquiatr. $v^{\mathbf{o}}$ 26, nº 5, 1999.

OJOPI, E. P. B., BERTONCINI, A. B., NETO, E. D. Apolipoproteína E e a doença de Alzheimer, Rev. Psiquiatr., v. 31, n. 1, p. 26 - 33, 2004.

SAMPAIO, H.A. de C.; SABRY, M. O. D. Nutrição em doenças crônicas: prevenção e controle. Atheneu, 2007.

SARAIVA, G. L. et al.Prevalência da deficiência de vitamina $\mathrm{D}$ e hiperparatiroidismo secundário em idosos institucionalizados e moradores na comunidade da cidade de São Paulo. Brasil. Arq. Bras. Endocrinol. Metab. v. 51, n. 3, p. 437 - 442. 2007.

SERENIKI, A., VITAL, M. A. Barbato Frazão. A doença de Alzheimer: aspectos fisiopatológicos e farmacológicos. Revista de Psiquiatria do Rio Grande do Sul. Porto Alegre, v. 30, n. 1, 2008. Disponível em: http://www.scielo.br/scielo.php?script=sci_art text\&pid=S0101$81082008000200002 \& \operatorname{lng}=$ pt\&nrm=iso. Acesso em: outubro de 2014.

SESHADRI, S. et al. Plasma homocysteine as a risk factor for dementia and Alzheimer's disease. The New England Journal of Medicine, v. 346, n. 7, p. 476-483, 2002.

SILVA, B. A. da; et al. Estado nutricional de idosos portadores do mal de Alzheimer. UNICRUZ, 2011.

SOUZA, M. et al. Risco Nutricional de Idosos com Doença de Alzheimer.

UNICRUZ, 2012.

TAVARES, E. L.; ANJOS, L. A. dos. Perfil antropométrico da população idosa brasileira. Resultados da Pesquisa Nacional sobre Saúde e Nutrição. Cad. Saúde Pública., v. 14, n. 4, p. $759-768,1999$.

VERAS, R. P.; RAMOS, L. R.; KALACHE, A. Crescimento da População Idosa no Brasil: Transformações e consequências na
Sociedade. Rev. Saúde Pública, n. 21, v. 3, p. $225-233.1987$. 